\title{
APPLYING PIV MEASUREMENTS ON FLOW FIELD OF WAVES PROPAGATING OVER A SINGLE SUBMERGED POROUS ELASTIC BREAKWATER
}

\author{
Tai-Wen Hsu ${ }^{1}$, Yuan-Jyh Lan ${ }^{1}$, Jian-Wu Lai ${ }^{1}$, Yung-Han Cheng ${ }^{1}$ and Shan-Hwei Ou ${ }^{2}$ \\ The objective of the present study is to investigate the flow field for waves propagating over a submerged poro-elastic \\ breakwater. Particle Image Velocimetry (PIV) measurements were performed in wave conditions with breakwaters \\ made of different materials. The experimental results were compared for various rigid and impermeable, elastic and \\ impermeable, as well as poro-elastic cases. Measurements of wave reflection and transmission induced by soft and \\ permeable submerged breakwaters are both carried out. The results show that the oscillatory motion of elastic \\ submerged breakwater can induce extra reflective waves and result in a larger reflection coefficient. Positive \\ (counterclockwise) and negative (clockwise) vortices are generated due to corner separation and pressure gradients. \\ The negative vortex obtained in a poro-elastic breakwater is generated by the surface friction at the top of the structure. \\ Because of the permeability, it is found that the negative vortex at the upstream side of the elastic and permeable case \\ is smaller than that of the rigid and impermeable one. In contrast, the positive vortex at the downstream side of the \\ poro-elastic case is larger than that seen with the other two cases. It is concluded that a poro-elastic breakwater would \\ induce different wave reflections and flow patterns from those seen with the other cases due to its particular wave and \\ structure interactions.
}

Keywords: poro-elastic structure; submerged breakwater; wave; PIV measurements

\section{INTRODUCTION}

Submerged breakwaters are structures that are set up in the surf zone to dissipate part of the sea's wave energy, reducing nearshore current velocity, and depositing sediments at leeward regions. If such structures are flexible, e.g. submerged flexible mounds (Ohyama et al., 1989) or a bed of large seagrasses (Stratigaki et al., 2011), the wave force will induce structural deformations. In addition, the subsequent movement of the structures will also affect the wave transformation and the flow field. Many researchers have examined rigid breakwaters with impermeable or permeable characteristics, and there are far fewer works that consider the interactions between waves and flexible structures. Wang and Ren (1993) proposed an analytical solution for the interaction of waves and a flexible, porous, and thin beam-like breakwater. This breakwater was conceptualized as a one-dimensional beam of uniform flexural rigidity and uniform mass per unit length, with the flow inside the porous structure obeying Darcy's law. Lan and Lee (2010) proposed an improved version of Biot's theory to evaluate highly permeable resistance, and analyzed the reflection, transmission and energy dissipation of monochromatic waves traveling over a single rectangular submerged poro-elastic structure. Based on the work of Lan and Lee (2010), Lan et al. (2011) obtained an analytical solution for Bragg scattering by a series of poro-elastic submerged structures with finite spacing, and the results were confirmed by laboratory experiments. In addition, the effects of seagrasses on unidirectional flows have been studied both numerically ( $\mathrm{Li}$ and Yan, 2007) and experimentally (Ghisalberti and Nepf, 2008). For example, Prinos et al. (2010) performed large scale experiments to measure wave attenuation, transmission and energy dissipation over artificial posidonia oceanica at intermediate and shallow water depths, while Stratigaki et al. (2011) experimentally investigated the effects of seagrass densities on wave attenuation and wave-induced flow within the meadow.

In recent years, due to the development of more sophisticated measuring techniques and computing capabilities, many researchers have applied flow visualization, Laser Doppler Velocimetry (LDV) and PIV for flow field measurements. Ting and Kim (1994) used a laser Doppler anemometer to observe the vortex phenomena in water waves passing over a rectangular submerged breakwater. Petti et al. (1994) applied PIV measurement to assess the flow field around a submerged breakwater, while Chang et al. $(2001,2005)$ used the same technique to measure the instantaneous flow field around a submerged rectangular obstacle with solitary and cnoidal waves. In the same study, a numerical model based on the two-dimensional Reynolds Average Navier Stokes (RANS) equations was proposed and tested with the experimental data. Lai et al. (2009) developed a motion visualization analysis system for laboratory observations based on a Charge Coupled Device (CCD) camera, and

\footnotetext{
${ }^{1}$ Dept. of Hydraulic and Ocean Engineering, National Cheng Kung University, 1 University Rd., Tainan, 701, TAIWAN (ROC)

${ }^{2}$ Dept. of Environmental Science and Occupational Safety and Hygiene, Tajen University, No.20,Weisin Rd., Sin-er Village,Yanpu, Pingtung, 907, TAIWAN (ROC)
} 
analyzed the wave characteristics and the motions of poro-elastic submerged breakwaters. Lai (2009) investigated the hydrodynamics and turbulence for waves propagating over a coarse-grained sloping beach using both experimental and numerical models, with both PIV and Digital Image Process (DIP) techniques applied in the laboratory experiment.

In this study, the PIV measurement system is employed to investigate the flow characteristics on waves propagating over a submerged poro-elastic breakwater. We analyze the measured instantaneous flow velocity around various types of structures by waves, and compare the results obtained for the vorticity field, including the traces of the vortex center and time history of maximum absolute vorticity. How the wave reflection and transmission are affected by breakwaters of various permeabilities and materials are also discussed.

\section{EXPERIMENTAL SETUP AND CONDITIONS}

The experiments for the present study were conducted in a glass-walled wave flume, which is $25 \mathrm{~m}$ long, $0.5 \mathrm{~m}$ wide and $0.6 \mathrm{~m}$ high. The details of the experimental arrangement are shown in Figure 1. The range of dashed square is the test section for observing wave and flow fields. A piston-type wavemaker is used, driven by an electromechanical hydraulic servo-system. At the end of the flume, a permeable slope with dissipative materials (tetrapod) is installed to keep the wave reflection coefficients at less than $10 \%$ of the wave height.

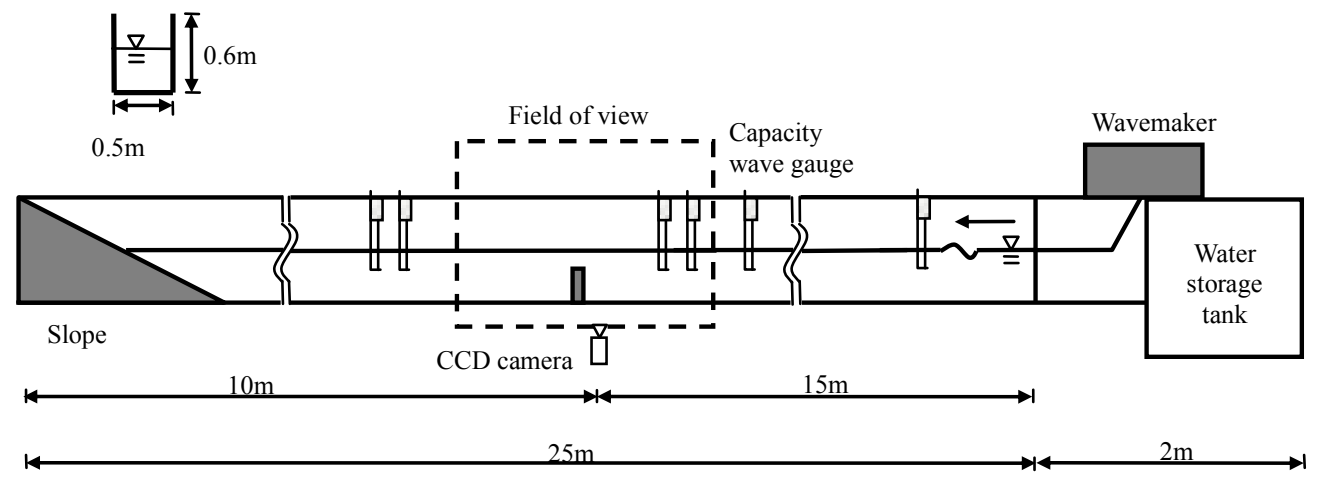

Figure 1. Schematic diagram of the experimental arrangement.

Figure 2 shows the experimental configuration of wave propagating over a submerged breakwater. A local Cartesian coordinate system for the experiment is used, with the origin located at the intersection of the flume bottom and the upstream side of breakwater, the $X$-axis pointed to the right, and the $Y$-axis pointed upward. The water depth $h$ is fixed at $21 \mathrm{~cm}$. The incident wave is specified as propagating in the $-X$ direction, with wave height $H=3 \mathrm{~cm}$. For wave reflection and transmission measurements, the wave period $T=0.6-2.0 \mathrm{~s}$, with an the interval of 0.1 seconds per test. The wave height was measured by using wave gauges. The three-gauge method proposed by Mansard and Funke (1980) was employed to calculate reflection coefficients and decompose the incident and reflected waves. In the PIV measurements of the flow field around the submerged breakwaters, two wave conditions were used in the experimental study, as shown in Table 1, with wave conditions of $T=1.0 \mathrm{~s}, k h=1.070$ ( $k$ is wave number) and $T=1.2 \mathrm{~s}, k h=0.849$. According to Muir Wood's (1969) scope classification in wave theory, the wave conditions listed in Table 1 represent linear waves of intermediate depths. 


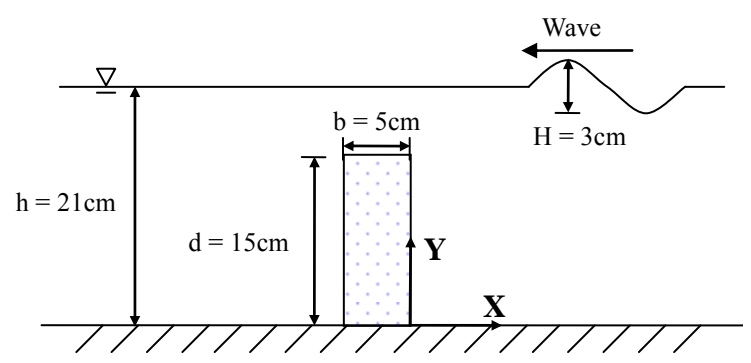

Figure 2. The sketch of experimental configuration of waves passing over submerged breakwater.

\begin{tabular}{|c|c|c|}
\hline \multicolumn{3}{|l|}{ Table 1. Wave conditions of laboratory experiments for flow fields. } \\
\hline Wave conditions & Case 1 & Case 2 \\
\hline$T(\mathrm{~s})$ & 1.0 & 1.2 \\
\hline$L(\mathrm{~m})$ & 1.233 & 1.553 \\
\hline$h(\mathrm{~m})$ & 0.21 & 0.21 \\
\hline$H(\mathrm{~m})$ & 0.03 & 0.03 \\
\hline $\mathrm{kh}$ & 1.070 & 0.849 \\
\hline
\end{tabular}

Analyses of the wave transformation and flow field in the experiment were undertaken using data measured during the 7 th to 11 th incident waves, in order to ensure the wave was developed and avoid the effects of the second reflection. In addition, the fluid velocity was obtained by averaging the results obtained from 30 repeated tests under the same conditions.

Three types of submerged structure are investigated in this study, and the physical properties are listed in Table 2. The structures have the same width $b=0.05 \mathrm{~m}$ and height $h=0.15 \mathrm{~m}$. Type (a) is a rigid and impermeable structure constructed of acrylic resin, with the shear modulus $G=5 \times 10^{9} \mathrm{~N} / \mathrm{m}^{2}$. Type (b) is an elastic and impermeable structure, made of a sponge-like material with waterproof treatment, and the shear modulus is $2.06 \times 10^{5} \mathrm{~N} / \mathrm{m}^{2}$. It is noted that, for the impermeable structures, the porosity $n^{\prime}$, intrinsic permeability $k_{p}$ and turbulent drag coefficient within structure $C_{f}$ are equal to zero. Type (c) is a the poro-elastic structure (sponge-like), for which the physical properties are $n^{\prime}=0.948, G=1.75 \times 10^{5} \mathrm{~N} / \mathrm{m}^{2}, k_{p}=7.432 \times 10^{-8} \mathrm{~m}^{2}$ and $C_{f}=8.195 \times 10^{-2}$.

\begin{tabular}{|c|c|c|c|}
\hline \multicolumn{5}{|l|}{ Table 2. Physical properties of submerged breakwaters. } \\
\hline Properties & Type (a) & Type (b) & Type (c) \\
\hline$b(\mathrm{~m})$ & 0.05 & 0.05 & 0.05 \\
\hline$d(\mathrm{~m})$ & 0.15 & 0.15 & 0.15 \\
\hline$n^{\prime}$ & 0 & 0 & 0.948 \\
\hline$G\left(\mathrm{~N} / \mathrm{m}^{2}\right)$ & $5 \times 10^{9}$ & $20.6 \times 10^{5}$ & $1.75 \times 10^{5}$ \\
\hline$k_{p}\left(\mathrm{~m}^{2}\right)$ & 0 & 0 & $7.432 \times 10^{-8}$ \\
\hline$C_{f}$ & 0 & 0 & $8.195 \times 10^{-2}$ \\
\hline
\end{tabular}

\section{ANALYSES METHOD}

The PIV technique, derived from flow visualization, is a new flow measurement technology. Using images of the flow field, the PIV technique can carry out quantitative calculations of the flow velocity. Consider that images of a fluid particle are obtained at two shooting locations at time $t_{1}$ and $t_{2}$ respectively, then the velocity of this particle $V_{i}$ is equal to $\Delta S / \Delta t$, where $\Delta S$ is the displacement of the particle from $t_{1}$ to $t_{2}$ and $\Delta t=t_{2}-t_{1}$. Based on this concept, each particle's velocity can be calculated theoretically. In order to distinguish all the particles relative to the fluid velocity in two successive images, statistical cross-correlation analysis on a pair of frames is employed in this study. The optimal particle displacement $(M, N)$ will appear in the case of the maximum corss-correlation coefficient. The actual velocity of fluid particles can thus be obtained by equation (1) 


$$
\overrightarrow{V_{R}}=M_{\text {opt }} \frac{M}{\Delta t} \vec{i}+M_{\text {opt }} \frac{N}{\Delta t} \vec{j}
$$

where $M_{\text {opt }}$ is the magnification rate between actual flow fields and images.

Four fields of views (FOVs) were used to capture the particles in the flow fields. Figure 3 shows the arrangement of FOVs. Points A, B and C are the checkpoints for checking the accuracy of the fluid velocity. In the PIV experiment, the camera aperture $f_{\#}=5.6$, lens magnification $M_{\text {opt }}=0.042$, laser wavelength $\lambda=532 \mu \mathrm{m}$, particle diameter $d_{p}=8 \mu \mathrm{m}$, and particle diameter in the images $d_{\tau}=7.58 \mu \mathrm{m}$. According to the uncertainty analysis carried out by Raffel et al (2007), the root-meansquare uncertainties in the experiment, in the interrogation windows of $32 \times 32$ and $64 \times 64$ pixel $^{2}$ are 0.1 and 0.023 pixel, respectively.

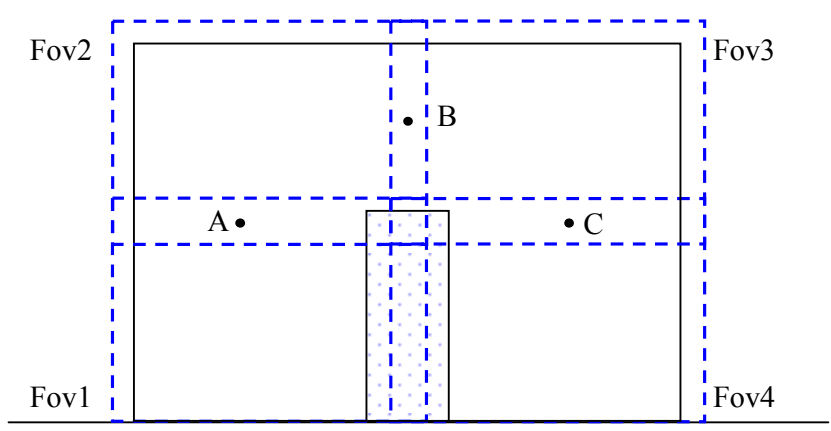

Figure 3. The arrangement of the field of views around the breakwater.

\section{RESULTS AND DISCUSSION}

The wave reflection and transformation, flow pattern, vortex vorticities and traces, as affected by the interaction of waves and poro-elastic submerged breakwaters made of different materials is discussed. The vorticity in the experimental study is defined as $\omega=\partial v / \partial X-\partial u / \partial Y$, where $u$ and $v$ are the horizontal and vertical components of fluid velocity, respectively. Positive vorticity represents a counter-clockwise vortex, and vice versa. The definition of the wave phase is illustrated in Figure 4. A wave period is accurately divided into ten phases by the synchronized control system, and phase $\boldsymbol{a}$ is defined as the time when the wave trough propagates over the upstream side of the submerged breakwater.

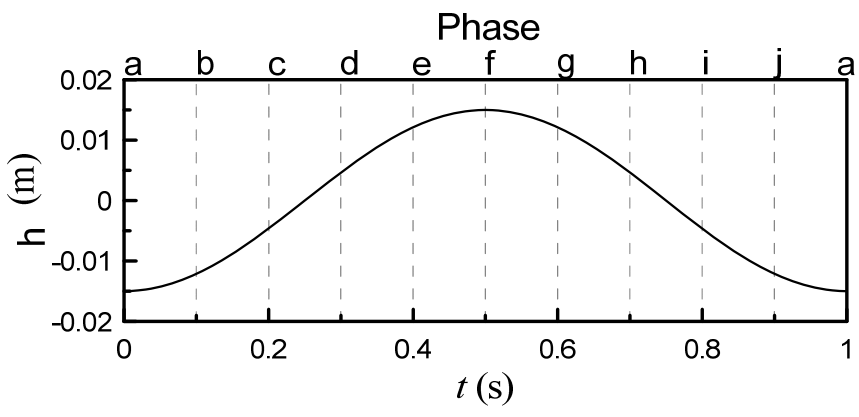

Figure 4. The arrangement of the wave phases.

\section{(a) Wave reflection and transmission}

Figure 5 shows the experimental results for the wave reflection coefficient $K_{R}$ and transmission coefficient $K_{T}$ versus $k h$ for waves propagating through the rigid impermeable, elastic impermeable and poro-elastic submerged breakwaters. It can be seen that the permeable submerged breakwater induces lower wave reflection than the impermeable ones. The wave energy dissipation that occurs with the permeable structure also decreases wave transmission. For the elastic and impermeable 
submerged breakwater, the wave-induced structural oscillations produce the effect of wave-making and increase wave reflection.

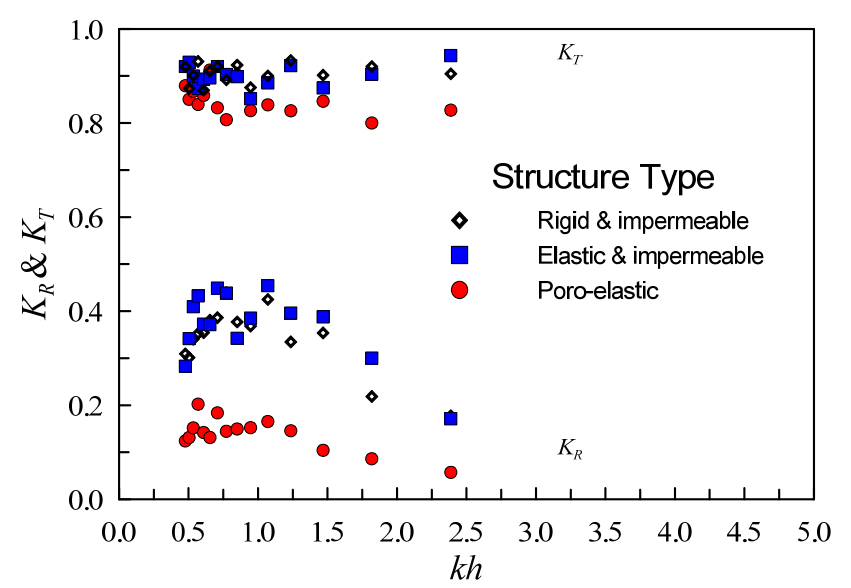

Figure 5. Comparisons of the three types of submerged breakwaters with regard to wave reflection coefficient $K_{R}$ and transmission coefficient $K_{T}$.

\section{(b) Flow field around the submerged breakwater $(T=1.0 \mathrm{~s})$}

This section discusses the flow fields around different types of submerged breakwaters. Figures 6 , 7 and 8 show the fluid velocity and vorticity fields around a submerged breakwater with rigid and impermeable, elastic and impermeable and poro-elastic properties, respectively, where the solid line represents the free surface elevation, and the dashed line represents the static water level. In the vorticity diagrams, the red areas with solid lines indicate a counterclockwise vortex (positive eddy vorticity), and the blue areas with dashed lines represent a clockwise vortex (negative eddy vorticity). For the case of the rigid and impermeable breakwater, Figure 6 reveals that a clockwise vortex exists near the upstream-top side of the submerged breakwater as a wave trough arrives at the structure (phase $\boldsymbol{a}$ ). In phase $\boldsymbol{b}$, the clockwise vortex gradually develops and separates from the upstream-top side. When the wave crest propagates near the upstream side of the breakwater (phases $\boldsymbol{c}$ and $\boldsymbol{d}$ ), the velocity direction of the flow field changes gradually from $+x$ to $-x$, and a counterclockwise vortex appears at top right corner of the breakwater and spreads along the top surface. Meanwhile, the clockwise vortex located at the upstream side moves up. The vorticity diagram in phase $\boldsymbol{e}$ shows that the clockwise vortex moves to the top right of the breakwater and the vorticity decreases. As the wave crest arrives at the rigid and impermeable submerged breakwater (phase $f$ ), the counterclockwise vortex region extends to leeward, and the clockwise vortex is taken to the fluid region above the submerged breakwater. After the wave crest passes through the submerged breakwater (phases $\boldsymbol{g}$ and h), a counterclockwise vortex in the downstream side separates and gradually grows while the clockwise vortex is almost totally dissipated. When the next wave trough approaches the submerged breakwater (phases $\boldsymbol{i}$ and $\boldsymbol{j}$ ), the flow direction gradually alters from the $-x$ to $+x$ direction, and the counterclockwise vortex disappears. After that, the wave motion finally returns to phase $\boldsymbol{a}$ to complete a cycle. Figure 7 illustrates the flow distribution around an elastic and impermeable submerged breakwater during a period of wave motion. It is noted that, in each phase, the relative positions of the vortexes appear similar to those seen for the rigid and impermeable structure shown in Figure 6. However, the vortex strength is disturbed due to the structural oscillation induced from the wave action. For the case of waves propagating over a poro-elastic submerged breakwater, Figure 8 reveals that both the effects of structural elasticity and permeability induce less fluid velocity near the top of the breakwater than the previous cases. In addition, as seen in phases $\boldsymbol{i}$ and $\boldsymbol{j}$, the structural permeability can lead the downstream-side vortex to a lower position from the top of the breakwater.

Figure 10 shows the details of the horizontal velocities at the profiles located $0.02 \mathrm{~m}$ from the breakwater surface as shown in Figure 9, in which the red lines indicate the top of the breakwater. We can see that the elastic effect of the submerged breakwater disturbs the flow distribution near and above the top of the breakwater. The permeability of material allows the fluid to pass through the submerged breakwater and flow downstream, and influences the horizontal velocity. 

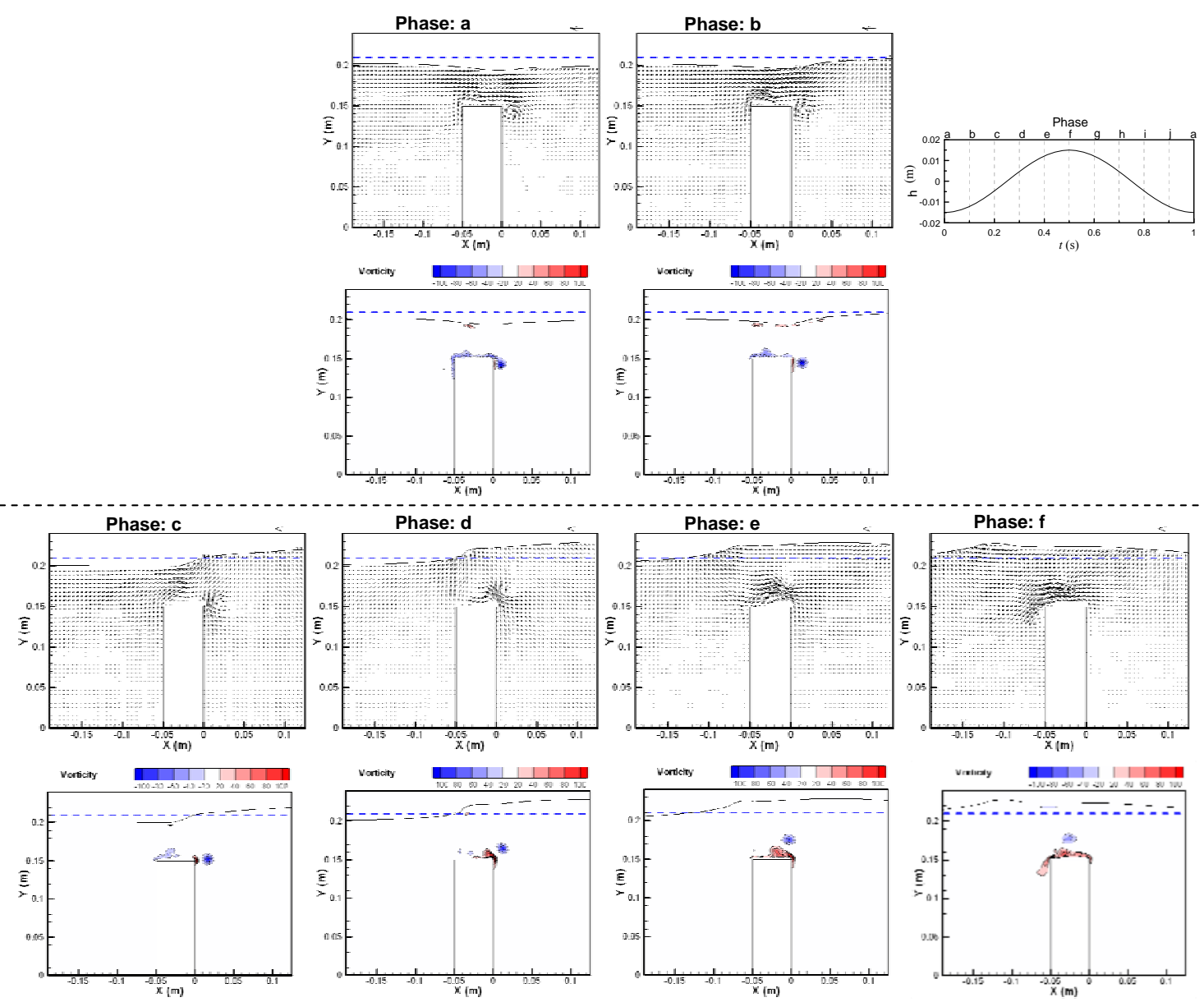

Phase: $g$
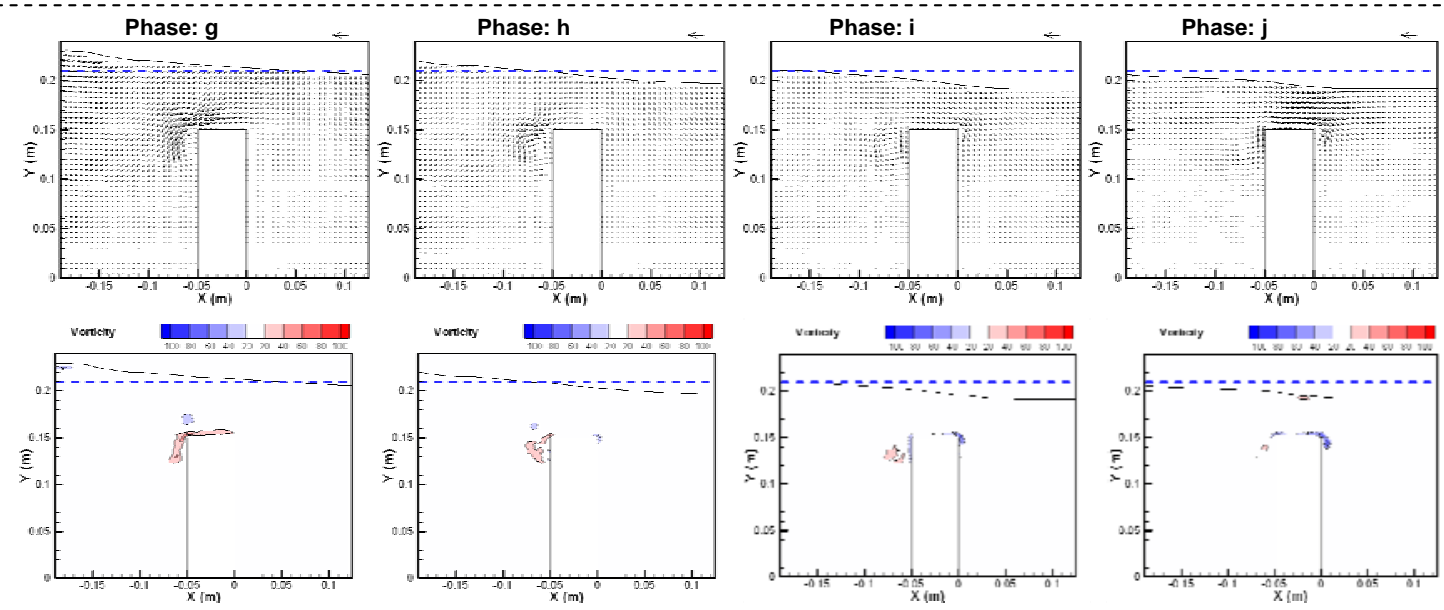

Figure 6. The measured velocity and vorticity fields around a rigid and impermeable submerged breakwater $(T=1.0 \mathrm{~s})$. 

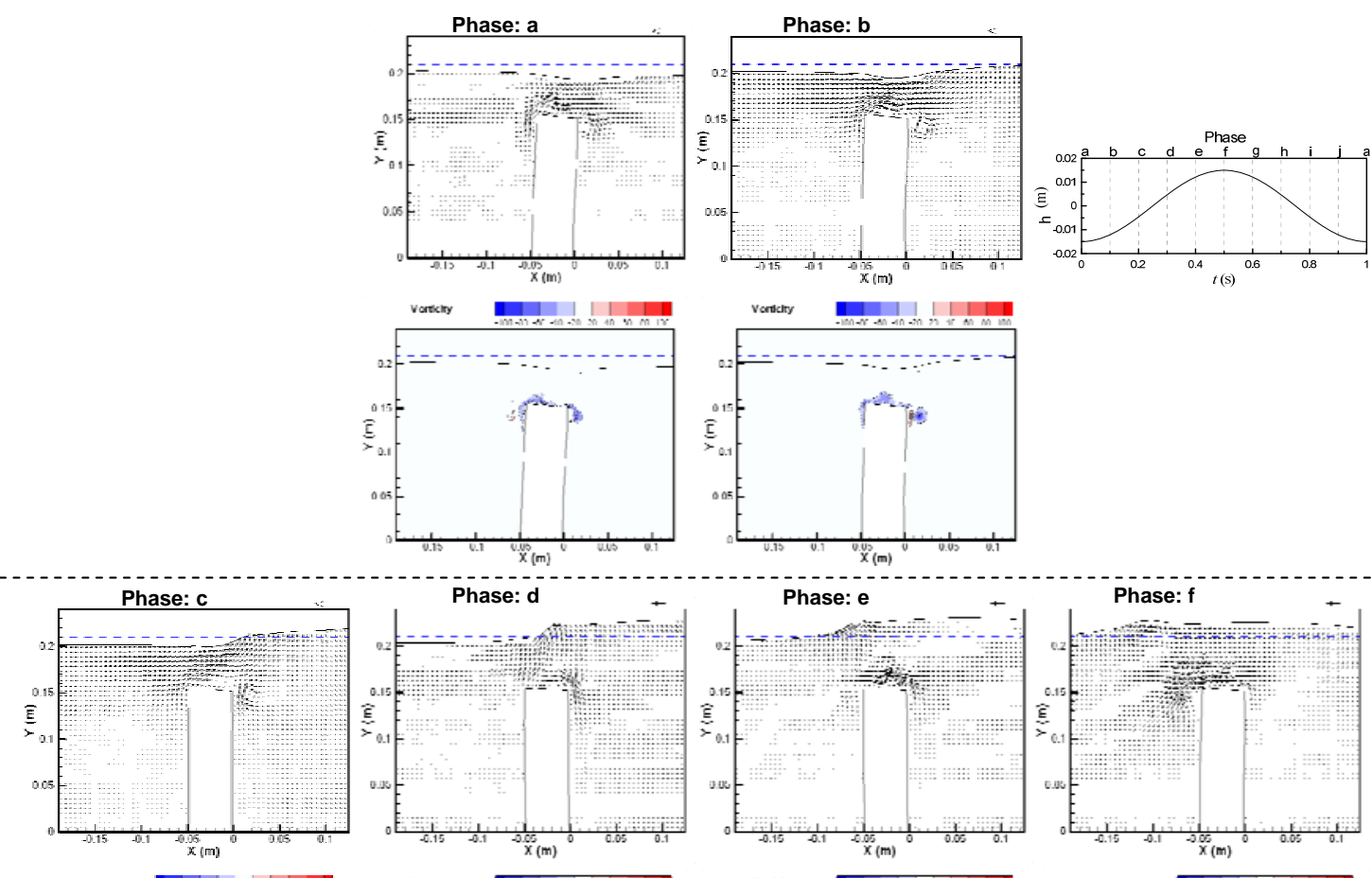

Phase: $f$
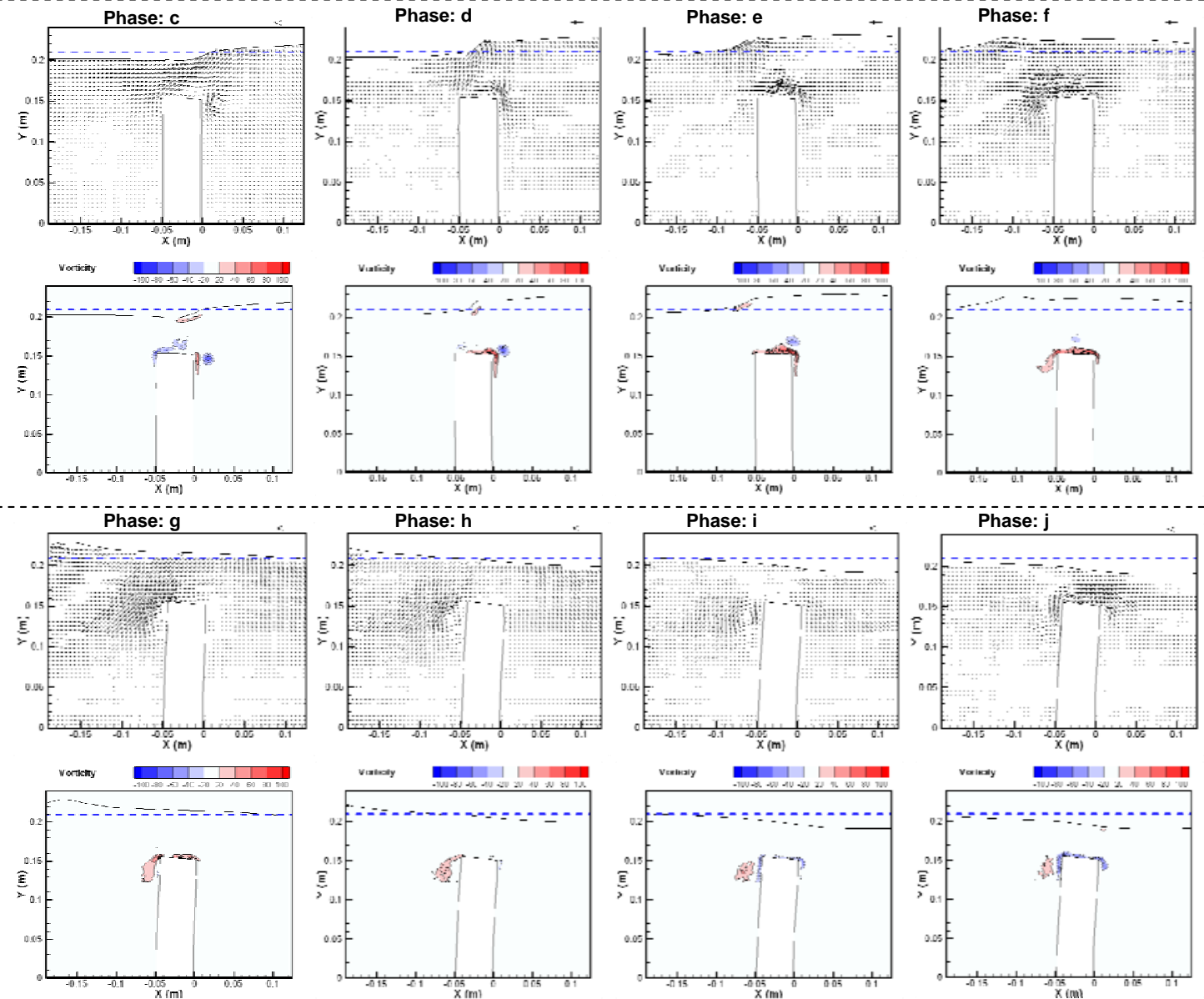

Figure 7. The measured velocity and vorticity fields around an elastic and impermeable submerged breakwater ( $T=1.0 \mathrm{~s}$ ). 

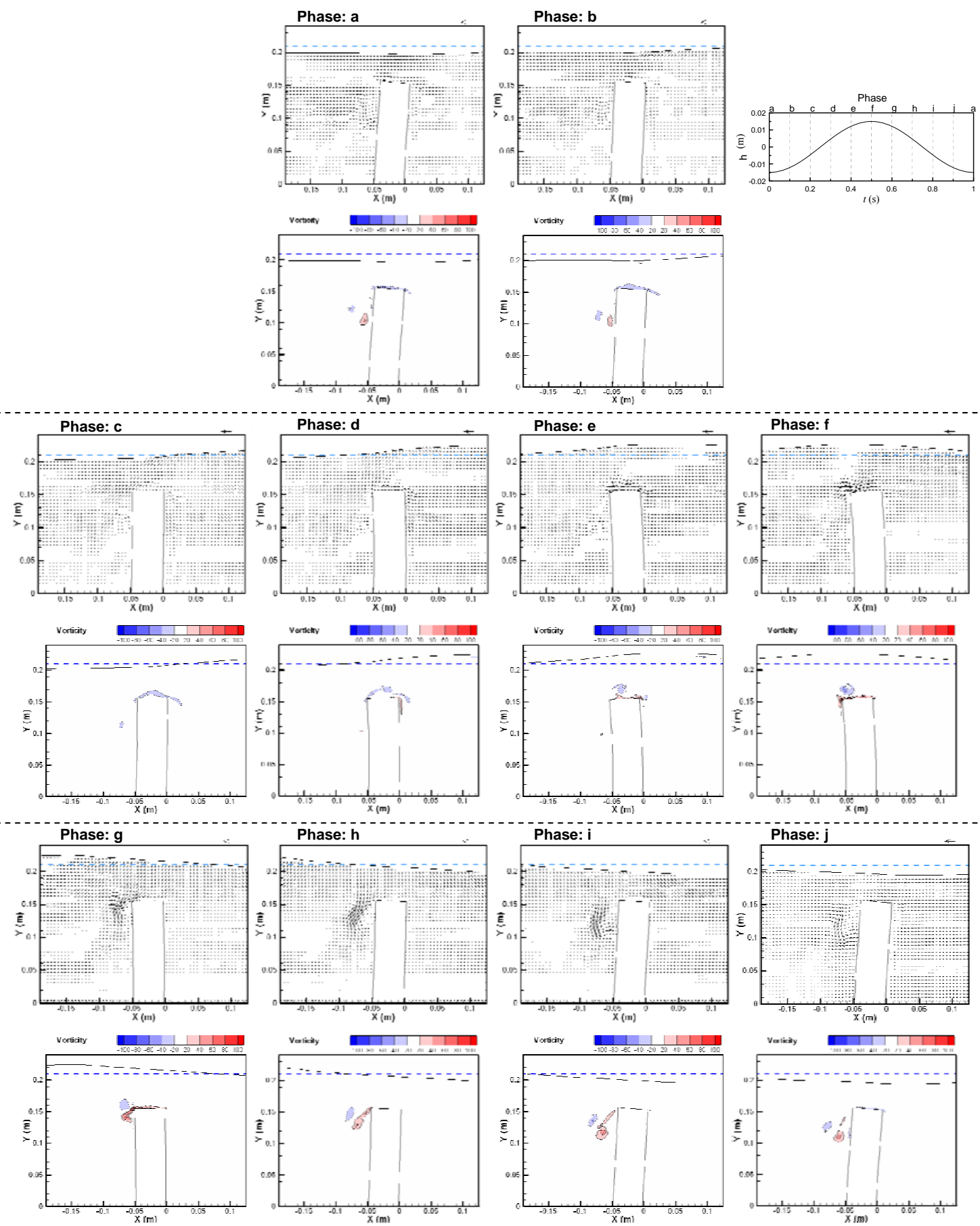

Figure 8. The The measured velocity and vorticity fields around a poro-elastic submerged breakwater ( $T=1.0 \mathrm{~s}$ ). 


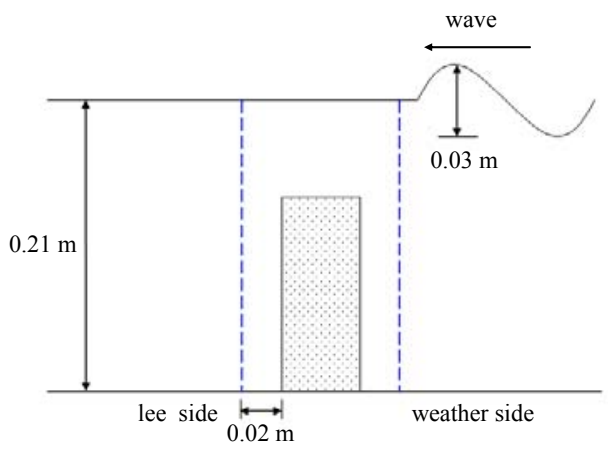

Figure 9. Sketch of the location for comparing the horizontal velocity profiles.

(a1) Downstream side; rigid and impermeable breakwater

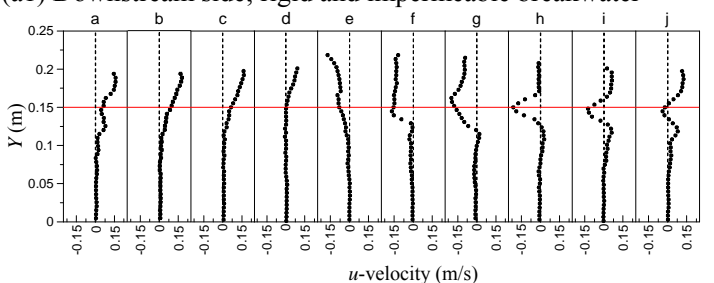

(b1) Downstream side; elastic and impermeable breakwater

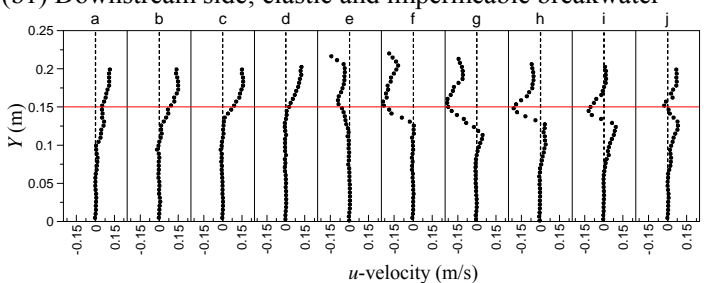

(c1) Downstream side; poro-elastic breakwater

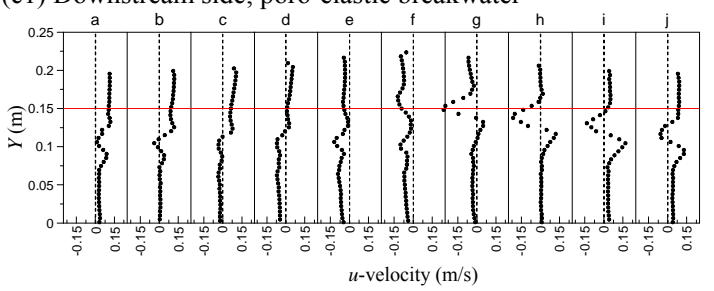

(a2) Upstream side; rigid and impermeable breakwater

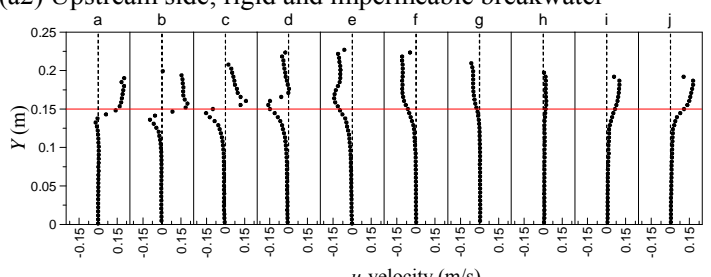

(b2) Upstream side; elastic and impermeable breakwater

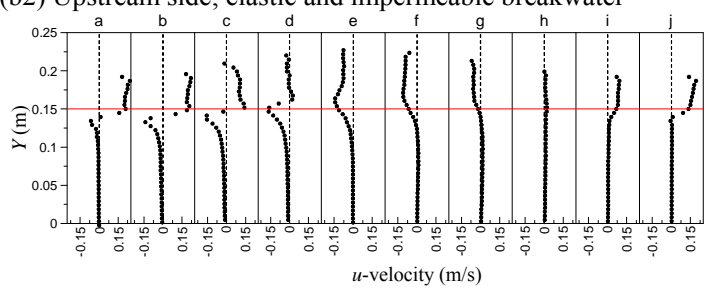

(c2) Upstream side; poro-elastic breakwater

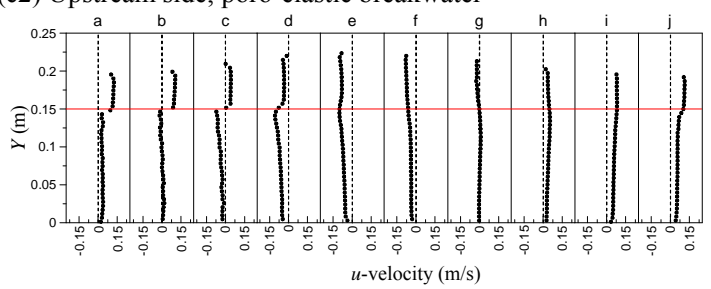

Figure 10. Comparisons of the horizontal velocity profiles during a wave period ( $T=1.0 \mathrm{~s}$; the profile positions are shown in Figure 9).

\section{(c) Traces of vortex center}

Figure 11 shows the traces of the vortex centers around the upper regions of the submerged breakwaters under the wave conditions of $T=1.0 \mathrm{~s}$ and $1.2 \mathrm{~s}$. In the figure, solid circles and lines represent the clockwise (negative) vortex, while hollow circles and dashed lines represent the counterclockwise (positive) vortex, red squares indicate the starting positions of vortex generation, and the letters represent the wave phases shown in Figure 4. The vortex center is defined as the place of the maximum absolute vorticity. The results show that the trace pattern in the elastic and impermeable case is similar to the condition of the rigid and impermeable breakwater. The clockwise vortex generates near the upstream-top side of the breakwater in wave phase $\boldsymbol{i}$, then crosses over the submerged breakwater to the downstream side and remains in the area above the breakwater. The counterclockwise vortex also generates at the upstream-top side of breakwater, and gradually moves to the downstream-top side. Due to the flexibility of the structure, the vortex center occurs at some distance from the upstream-top corner of the elastic and impermeable breakwater. As for the poroelastic submerged breakwater, its permeability causes the clockwise vortex to occur at the top of the breakwater in wave phase $\boldsymbol{c}$, then shifting over the breakwater, move down to the downstream region, 
and dissipate. The counterclockwise vortex is confined to the fluid field of the downstream side, generating near the downstream-top corner of the breakwater in wave phase $f$, and then gradually moving toward the under direction.
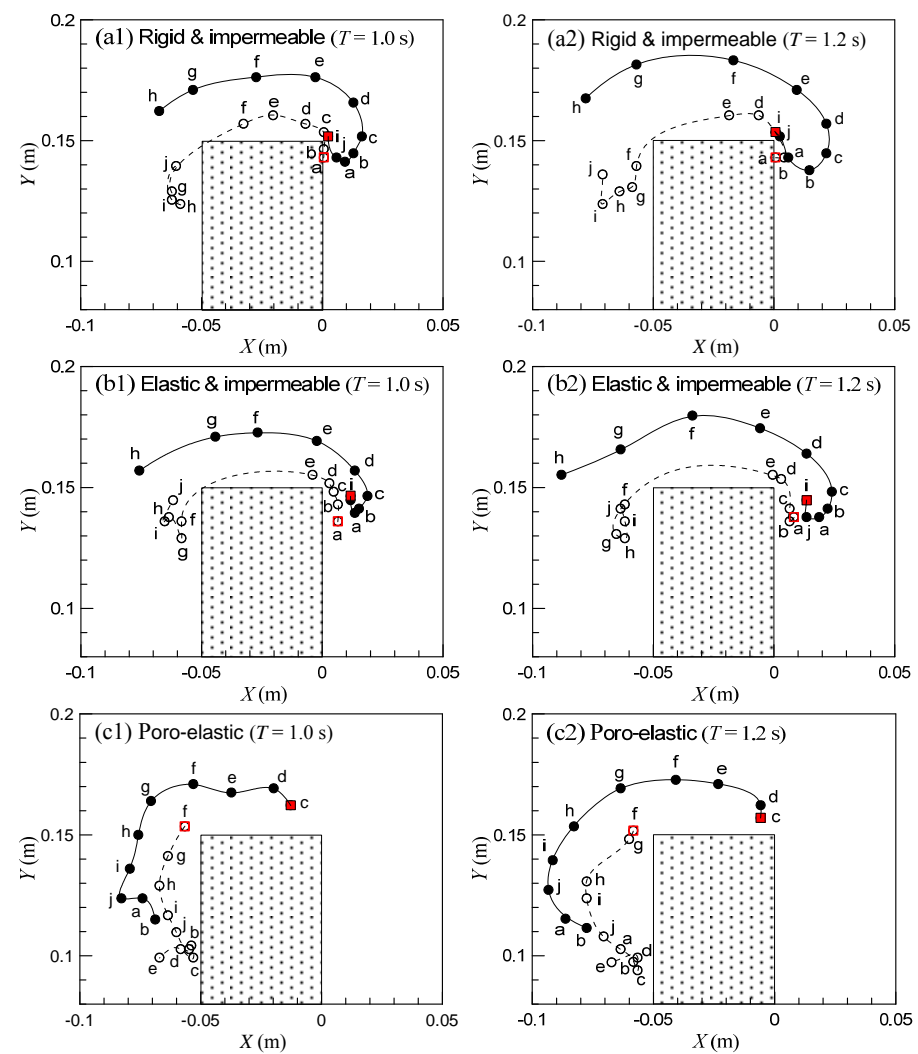

Figure 11. Comparisons for the traces of vortex centers during a wave period (O: Negative (clockwise) vortex, $\bigcirc:$ Positive (counterclockwise) vortex).

\section{(d) Maximum absolute vorticity}

Figure 12 shows the time history of maximum absolute vorticity $\omega_{\max }$ in the wave with period of $1.0 \mathrm{~s}$ and $1.2 \mathrm{~s}$ propagating through rigid and impermeable, elastic and impermeable, and poro-elastic submerged breakwaters. From the results in Figures 12(a1) 12(b2), we can find the maximum vorticity $\omega_{\max }$ of the clockwise vortex induced by the elastic and impermeable submerged breakwater is smaller than that for the rigid and impermeable case. At $t / T=0.6 \sim 0.9$ (phases $\boldsymbol{g}$ to $\boldsymbol{j}$ ), the oscillation of the elastic and impermeable structure increases the vorticity $\omega_{\max }$ of the counterclockwise vortex. For the case of the poro-elastic submerged breakwater, comparing Figures 12 (b1) 12(c2), it is found that the structural permeability decreases the difference in fluid velocity around the upstream-top corner of the breakwater and reduces the clockwise vorticity, while the counterclockwise vortex increases at $t / T=0.5 \sim 0.9$ (phases $\boldsymbol{f}$ to $\boldsymbol{j}$ ). 

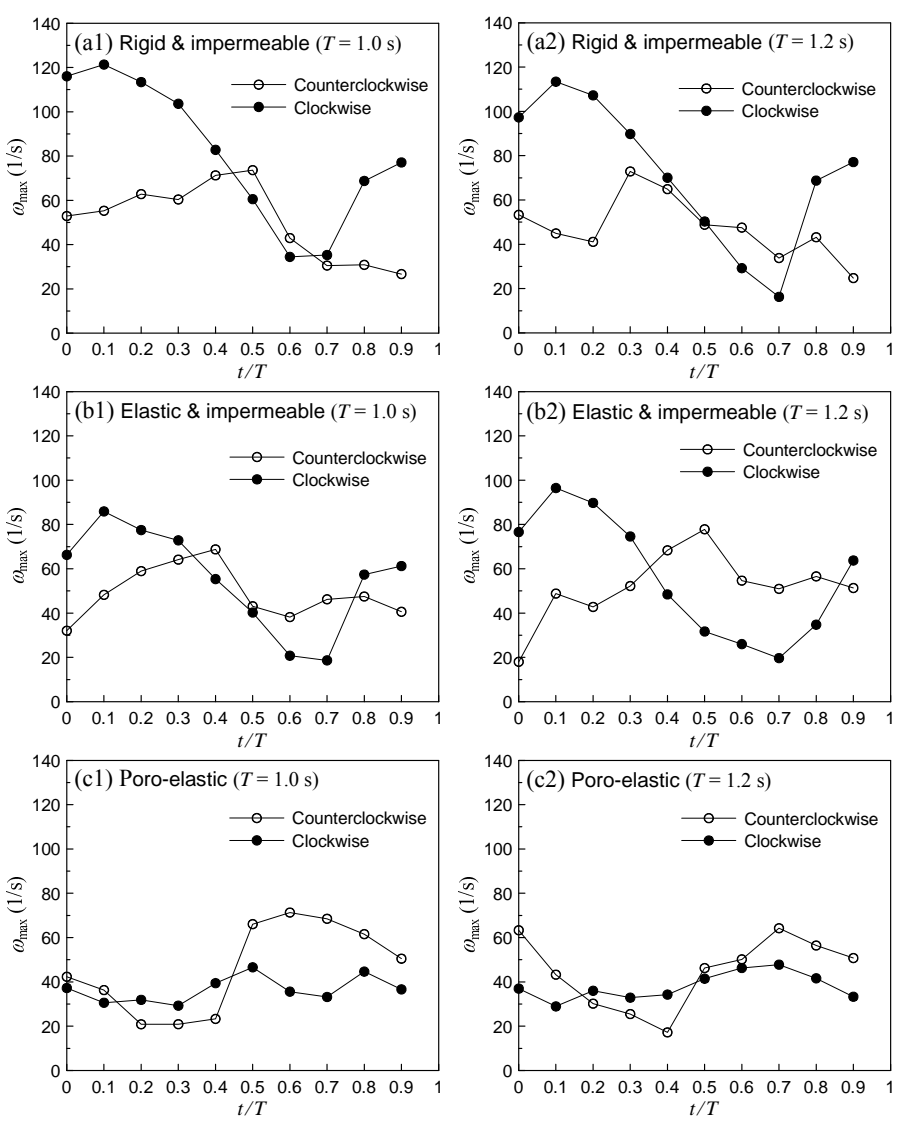

Figure 12. Time history of maximum absolute vorticity (counterclockwise) vorticity).

\section{CONCLUSION}

PIV measurements and experimental analyses were performed to examine waves propagating over a submerged porous elastic breakwater. Using the global average method to analyze the data from 30 repeated tests, the characteristics of wave reflection, transmission, flow field and vortex were investigated and discussed. Based on the results, the following findings are presented. The waveinduced oscillations from the elastic and impermeable submerged breakwater produce waves in the wave field and increase the reflection coefficient. For the poro-elastic submerged breakwater, the permeability induces less wave reflection, and the wave energy dissipation by permeable effect also decreases wave transmission. Because of the permeability, the clockwise vortex at the upstream side of the elastic and permeable structure is smaller than that of rigid and impermeable case. In contrast, the counterclockwise vortex at the leeward side for poro-elastic case gradually becomes larger than that of the other two cases. It is concluded that a poro-elastic breakwater would induce different wave reflections and flow patterns due to its particular wave and structure interactions.

\section{ACKNOWLEDGMENTS}

This study was financially supported by the National Science Council of Taiwan under grant numbers NSC96-2221-E-006-348-MY2 and NSC100-2221-E-006-010-MY3.

\section{REFERENCES}

Chang, K.A., T.J. Hsu, and P.L.F. Liu. 2001. Vortex generation and evolution in water waves propagating over a submerged rectangular obstacle, part I. solitary waves, Coastal Engineering, 44, 13-36.

Chang, K.A., T.J. Hsu, and P.L.F. Liu. 2005. Vortex generation and evolution in water waves propagating over a submerged rectangular obstacle, part II: cnoidal waves, Coastal Engineering, $52,257-283$. 
Ghisalberti, M., and H.M. Nepf. 2008. Sallow flows over a permeable medium: The hydrodynamics of submerged aquatic canopies, Transport in Porous Media, 78, 385-403.

Lai, J.W. 2009. Digital image technique and data application on monitoring water surface in wave flume, Ph.D. Thesis, National Cheng Kung University, Taiwan, ROC.

Lai, J.W., Y.J. Lan, T.W. Hsu, C.H. Ting, and C.C. Chang. 2009. Application of digital image process on observing the motions of wave and a series of poro-elastic submerged breakwaters, Proceedings of Coastal Dynamics 2009, paper No. 142.

Lan, Y.J., and J.F. Lee. 2010. On Waves Propagating over a Submerged Poro-Elastic Structure, Ocean Engineering, 37, 705-717.

Lan, Y.J., T.W. Hsu, J.W. Lai, C.C. Chang, and C.H. Ting. 2011. Bragg scattering of waves propagating over a series of poro-elastic submerged breakwaters, Wave Motion, 48, 1-12.

Li, C.W., and K. Yan. 2007. Numerical investigation of wave-current-vegetation interaction, Journal of Hydraulic Engineering, 133, 794-803.

Mansard, E.P.D., and E.R. Funke. 1980. The measurement of incident and reflected spectra using a least squares method, Proceedings of the 17th International Conference on Coastal Engineering, ASCE, 2, 154-172.

Muir Wood, A.M. 1969. Coastal hydraulics, New York :Gordon and Breach, 187p.

Ohyama, T., M. Tanaka, T. Kiyokawa, T. Uda, and Y. Murai. 1989. Transmission and reflection characteristics of waves over a submerged flexible mound, Coastal Engineering in Japan, 32(1), 53-68.

Petti, M., P.A. Quinn, G. Liberatore, and W.J. Easson. 1994. Wave velocity field measurement over a submerged breakwater. Proceedings of the 24th International Conference on Coastal Engineering, ASCE, 525-539.

Prinos, P., V. Stratigaki, E. Manca, I. Losada, J.L. Lara, M. Sclavo, I. Cáceres, and A. Sánchez-Arcilla. 2010. Wave propagation over Posidonia oceanica: large scale experiments, Proceedings of the HYDEALAB III Joint User Meeting, Hannover.

Raffel, M., C. Willert, S. Wereley, and J. Kompenhans. 2007. Particle image velocimetry : A practical guide, 2nd ed. Springer.

Stratigaki, V., E. Manca, P. Prinos, I. Losada, J.L. Lara, M. Sclavo, C.L. Amos, I. Cáceres, and A. Sánchez-Arcilla. 2011. Large-scale experiments on wave propagation over posidonia oceanica, Journal of Hydraulic Research, 49(S1), 31-43.

Ting, F.C.K., and Y.K. Kim. 1994. Vortex generation in water waves propagation over a submerged obstacle, Coastal Engineering, 24, 23-49.

Wang, K.H., and X. Ren. 1993. Water waves on flexible and porous breakwaters, Journal of Engineering Mechanics, 119, 1025-1047. 